\title{
Arbeitsmarktpolitik aus der "Mottenkiste"? Kurzarbeitergeld im Lichte politischer Interessen
}

Infolge der Wirtschaftskrise stand in Deutschland ein arbeitsmarktpolitisches Instrument besonders im Zentrum - das Kurzarbeitergeld. Dieser Klassiker der Arbeitsmarktpolitik wurde in seiner Geschichte immer dann vermehrt genutzt, wenn - wie auch im aktuellen Abschwung geschehen - seine Regulierung so modifiziert wurde, dass der Einsatz für Arbeitgeber an Attraktivität gewann. Es ist daher nicht allein die Konjunktur, die den Mitteleinsatz antreibt. Der Einfluss entsprechender politischer Steuerung sollte nicht unterschätzt werden. ${ }^{1}$

\section{Griff in die Mottenkiste}

Infolge der globalen Finanz- und Wirtschaftskrise brach das Wirtschaftswachstum in Deutschland im Verlauf des Jahres 2008 stärker als in anderen europäischen Ländern ein. Überraschenderweise reagierte der deutsche Arbeitsmarkt vergleichsweise robust (Stein/Aricò in diesem Heft). Im Fokus des wissenschaftlichen und öffentlichen Interesses stand daher das Kurzarbeitergeld (KUG), das zum deutschen „Beschäftigungswunder“2 beigetragen hatte. Spielte das konjunkturelle KUG als arbeitsmarktpolitisches Instrument im vorangegangenen Abschwung praktisch keine Rolle mehr, so stieg in der aktuellen Finanz- und Wirtschaftskrise die Zahl der KUG-Empfänger deutlich an (Abbildung 1). Bereits in früheren Krisen nutzte man das KUG zum Aufbau einer Beschäftigungsbrücke, der bildlich gesprochen die Funktion zukommt, bei der Überbrückung des temporär bestehenden Konjunkturtals zu helfen. Neben dem KUG können verschiedene Instrumente der intern-numerischen Flexibilität genutzt werden, um Entlassungen zu vermeiden oder in ihrem Ausmaß einzuschränken. Eichhorst/Marx (2009) gehen davon aus, dass durch eine gestiegene Flexibilisierung der Arbeitszeiten in Tarifverträgen und Betriebsvereinbarungen Kurzarbeit an Bedeutung verloren hätte. Analysen zum jüngsten Abschwung zeigen jedoch, dass diese neuen Instrumente nicht substitutiv, sondern komplementär genutzt werden können (HerzogStein/Seifert in diesem Heft; Bogedan, C./ Brehmer, W./Herzog-Stein, A. 2009). So standen im aktuellen Abschwung den Betrieben mit Kurzarbeit, Arbeitszeitkonten und betrieblichen Arbeitszeitverkürzungen gleich drei Pfeiler zur Verfügung, die der Beschäftigungsbrücke einen stabilen Halt geben konnten. Die konkrete Bedeutung dieser drei Instrumente variierte natürlich je nach Einzelfall (Heckmann et al. 2009); dort wo Beschäftigungssicherung stattfand, kamen diese Maßnahmen in unterschiedlicher Kombination zum Einsatz. Neben der Beschäftigungssicherung erfolgte auch Beschäftigungsabbau (Bogedan/Brehmer/Herzog-Stein 2009; Heckmann et al. 2009). Der Einsatz des KUG als Kriseninstrument wurde von der damaligen Bundesregierung Ende 2008 forciert, indem sie den Zugang erleichterte, die Bezugsdauer verlängerte und die Sozialversicherungsbeiträge übernahm. Wie kam es zu dieser Renaissance eines tot geglaubten Instruments?

Die Motive von Politik, Arbeitgebern (AG) und Arbeitnehmern (AN), KUG in diesem Abschwung intensiv zu nutzen, sind Gegenstand der vorliegenden Untersuchung. Der Beitrag analysiert hierzu zunächst die Funktionsweise des KUG (Abschnitt 2). Es zeigt sich, dass die Regulierung des KUG an sich wenig Anreize für die Betriebe zu seiner Nutzung bietet. Die Politik befindet sich folglich in einem Dilemma: Will sie KUG zur Beschäftigungssicherung in einer Krise nutzen, muss sie die Regulierung des KUG generöser gestalten. Dies ist ohne einen intensiven Mitteleinsatz und die Gefahr möglicher Trittbrettfahrer nicht möglich (Brenke/ Zimmermann 2010). Denn je generöser die Ausgestaltung, desto eher werden auch Betriebe das KUG nutzen, deren Arbeitsausfall nicht unvermeidbar war. Im Rahmen eines historischen Vergleichs wird in einem zweiten Schritt analysiert, unter welchen Bedingungen die Politik in Deutschland eine solche Ausweitung dennoch prakti- zierte (Abschnitt 3 und 4). Abschnitt 5 umreißt zusammenfassend politische Konsequenzen und Forschungsbedarf.

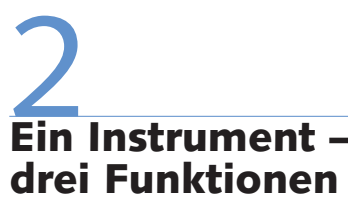

Die Sicherung von Beschäftigungsverhältnissen gehört in einem konjunkturellen Abschwung zu den vorrangigen arbeitsmarktpolitischen Aufgaben. Das KUG hilft, bei einem vorübergehenden Arbeitsausfall Arbeitsplätze zu sichern. Es kann daher grundsätzlich von Betrieben beantragt werden, wenn aufgrund wirtschaftlicher Ursachen oder besonderer unabwendbarer Ereignisse ein voraussichtlich vorübergehender Arbeitsausfall droht. AuBerdem müssen folgende Voraussetzungen erfüllt sein: Der Entgeltausfall muss min-

1 Die Autorin dankt dem Heftkoordinator, den anderen Autorinnen und Autoren des Schwerpunktheftes sowie den Kolleginnen und Kollegen der ESPAnet Konferenz 2010 für hilfreiche Kommentare und Anregungen. Christopher Lehmann, Matthias Klein, Susanne Schulz und Barbara Zimmer sei gedankt für die Unterstützung bei der Datenrecherche und Korrektur.

2 Paul Krugmann beschrieb „Germany's jobs miracle $^{\prime \prime}$ in seiner Kolumne für die New York Times vom 17. November 2009. Auch der Economist griff dieses Bild in seinem vom 13.-19.03.2010 erschienenen "special report on Germany“ auf.

Claudia Bogedan, ist Wissenschaftlerin im Wirtschafts- und Sozia/wissenschaftlichen Institut (WSI) in der Hans-Böckler-Stiftung. Arbeitsschwerpunkte: International verg/eichende Arbeitsmarktpolitik, soziale Sicherung flexibler Erwerbsbiografien. e-mail: claudia-bogedan@boeckler.de 
destens 10 \% für ein Drittel der Beschäftigten betragen. Es müssen zuvor alle anderen Möglichkeiten, den Arbeitsausfall abzufedern (z.B. Nutzung von Tarifvereinbarungen, Abbau von Arbeitszeitkontenguthaben), ausgeschöpft worden sein. Die AN müssen ein sozialversicherungspflichtiges Beschäftigungsverhältnis haben und dies auch fortsetzen. Das KUG ersetzt für den Arbeitsausfall einen Teil des entgangenen Nettolohns (derzeit $60 \%$ für Kinderlose, 67 \% für KUG-Empfänger mit Kindern). Die reguläre maximale Bezugsdauer beträgt sechs Monate. Diese kann aber durch Rechtsverordnung des Ministers verlängert werden, wenn „außergewöhnliche Verhältnisse in bestimmten Wirtschaftszweigen oder Bezirken vorliegen" (Schmid/ Semlinger 1980).

Dem KUG kommt damit-ähnlich wie dem Arbeitslosengeld - eine doppelte Rolle zu: Einerseits schützt es den individuellen AN vor Einkommensausfällen und andererseits wirkt es auf der Makroebene als automatischer Stabilisator, indem (drastische) Einkommens- und Konsumausfälle vermieden werden. Das KUG ist folglich vom Wesen her eine wirtschafts- wie eine arbeitsmarktpolitische Leistung, aber eben auch in ihrer Einkommensersatzfunktion eine klassische Versicherungsleistung (Schmid/Semlinger 1980). Diese unterschiedlichen Funktionen des KUG (Mosley/Kruppe 1995) haben sich seit 1970 in einer Ausdifferenzierung des Instruments in Konjunktur-, Transfer- und Saison-KUG niedergeschlagen.

Dabei wirkt das KUG über drei Mechanismen: Erstens wird ein Teil des durch den Arbeitsausfall begründeten Verdienstausfalls für den AN ausgeglichen. Zweitens kann der AG den Arbeitsausfall auf mehrere Schultern verteilen und somit Entlassungen und die damit verbunden Kosten vermeiden. Drittens vermeidet der Staat bzw. die Bundesagentur für Arbeit die andernfalls anfallenden Kosten für Arbeitslosigkeit (Bruche/Reissert 1985). Für die Betriebe ist dieses „Halten“ von Beschäftigten umso interessanter, je betriebsspezifischer die notwendigen Qualifikationen sind. Da für Betriebe allerdings ein Teil der Lohnnebenkosten auch für die Ausfallzeit zu tragen ist, lohnt die Kurzarbeit nur solange, wie diese sogenannten Remanenzkosten der Kurzarbeit kleiner sind als die Humankapitalverluste und Wiedergewinnungskosten bei Entlassungen (Bach/ Spitznagel 2009). Die mögliche Dauer des
KUG-Bezuges ist daher ein wichtiger Parameter zur Bewertung des Nutzens des Instruments (Crimmann et al. 2009). Allerdings sind die Kosten eines Personalwechsels kaum generell zu quantifizieren. Man kann jedoch davon ausgehen, dass es mit zunehmender Dauer der Krise immer schwieriger für die Betriebe wird, die Beschäftigten zu halten (Bach et al. 2009; Haag/Discher 2002).

Allen drei Parteien, Arbeitnehmern, Arbeitgebern und Staat, kann daher unterstellt werden, dass sie ein Interesse haben, Beschäftigung zu sichern: sicherlich am stärksten für den Fall einer Konjunkturkrise und eines damit verbundenen vorübergehenden Nachfrageausfalls, was der ursprünglichen Konzeption des KUG entsprach. Aber auch bei strukturellen Unternehmens- oder Betriebskrisen kann Beschäftigungssicherung sinnvoll sein, wenn beispielsweise erwartet wird, dass nach der Umstrukturierung wieder mehr Arbeitskräfte benötigt werden. AN haben auch dann Interesse an Beschäftigungssicherung, wenn aufgrund von Rationalisierung Beschäftigungsabbau droht und die allgemeine Lage am Arbeitsmarkt eine zeitnahe oder vergleichbare Weiterbeschäftigung erschwert. In diesen Fällen kann Kurzarbeit mit den entsprechenden Begleitmaßnahmen (wie Weiterbildung) dazu beitragen, die Beschäftigungsfähigkeit zu verbessern und die Suchphase zu überbrücken. Aus Perspektive der AN kann bestenfalls Arbeitslosigkeit verhindert und andernfalls das Eintreten der Arbeitslosigkeit sozial abgefedert werden. Die „Kosten“ der Kurzarbeit sind zudem ungleich zwischen den drei beteiligten Akteuren - AN, AG und Staat - verteilt (Flechsenhar 1978).

\section{3 KUG im Konjunkturverlauf}

Mit der Modifikation der KUG-Regulierung erlebte 2009 ein nahezu tot geglaubtes Instrument der aktiven Arbeitsmarktpolitik eine unverhoffte Renaissance. Bereits in der ersten großen Rezession der Nachkriegsgeschichte in den 1970er Jahren nutzte man das KUG mit dem Ziel, eine Brücke über das Konjunkturtal zu schlagen und Arbeitsplätze zu erhalten. In der Arbeitsmarktkrise nach der Wiedervereinigung in den 1990er Jahren griff man erneut auf das KUG zurück, da es sich recht voraussetzungslos und schnell auch in den neuen Ländern implementieren ließ. Abbildung 1 zeigt deutlich, dass seit 1970 die Zahl der Empfänger von KUG zu vier Zeitpunkten besonders hoch war (1975, 1983, 1991 und 2009). Hier betrugen die Ausgaben für Kurzarbeit, gemessen an den Gesamtausgaben der Bundesagentur für Arbeit (BA), annähernd $10 \%$ und mehr, in den anderen Jahren lagen sie deutlich darunter.

Die Entwicklung der KUG-Empfänger sowie der Verlauf der anteiligen Ausgaben für Kurzarbeit an den Ausgaben der Arbeitsmarktpolitik verlaufen entgegengesetzt zu den jährlichen Wachstumsraten des Bruttoinlandsproduktes (BIP). Zu fünf Zeitpunkten in den vergangenen vier Jahrzehnten fiel das BIP: 1975, 1982, 1993, 2003 und 2009. Diese Zeitpunkte sind zwar nicht mit den Spitzen bei der Zahl der KUG-Empfänger identisch, liegen aber in zeitlicher Nähe. Konjunktureinbrüche treiben folglich die Nutzung des KUG an. Nutzt man statt des BIP-Wachstums einen „sensibleren“ Maßstab für die wirtschaftliche Entwicklung, lassen sich die verschiedenen Abschwünge besser abgrenzen. Dies ermöglicht das Konzept der Outputlücke, d.h. die prozentuale Abweichung des BIP vom Produktionspotenzial. Damit lässt sich nach der Definition des Sachverständigenrats zur Begutachtung der gesamtwirtschaftlichen Entwicklung (SVR 2007) Beginn und Ende eines bedeutenden konjunkturellen Auf- bzw. Abschwungs bestimmen. Herzog-Stein/ Seifert (2010) identifizierten auf dieser Basis fünf relevante Abschwünge: (1) 2. Quartal 1973 - 2. Quartal 1975, (2) 4. Quartal 1979 - 4. Quartal 1982, (3) 1. Quartal 1991 - 3. Quartal 1993, (4) 1. Quartal 2001 2. Quartal 2005, (5) 1. Quartal 2008 2. Quartal 2009. Es zeigt sich, dass sowohl die Entwicklung der Kurzarbeit als auch das negative BIP-Wachstum jeweils nach Beginn des jeweiligen Abschwungs lagen. Zudem ist zu erkennen, dass sich die Abschwünge in ihrer Dauer und Heftigkeit deutlich unterscheiden. So fällt beispielsweise der Abschwung nach der Wiedervereinigung, was das Zusammenspiel von Einbruch des BIP-Wachstums und Zahl der KUG-Empfänger betrifft, aus dem allgemeinen Muster.

Ein weniger zyklisches Muster zeigt die Entwicklung der Arbeitslosigkeit. Dies liegt vor allem daran, dass es auch in 


\section{Abb. 1: Kurzarbeit und Konjunktur}

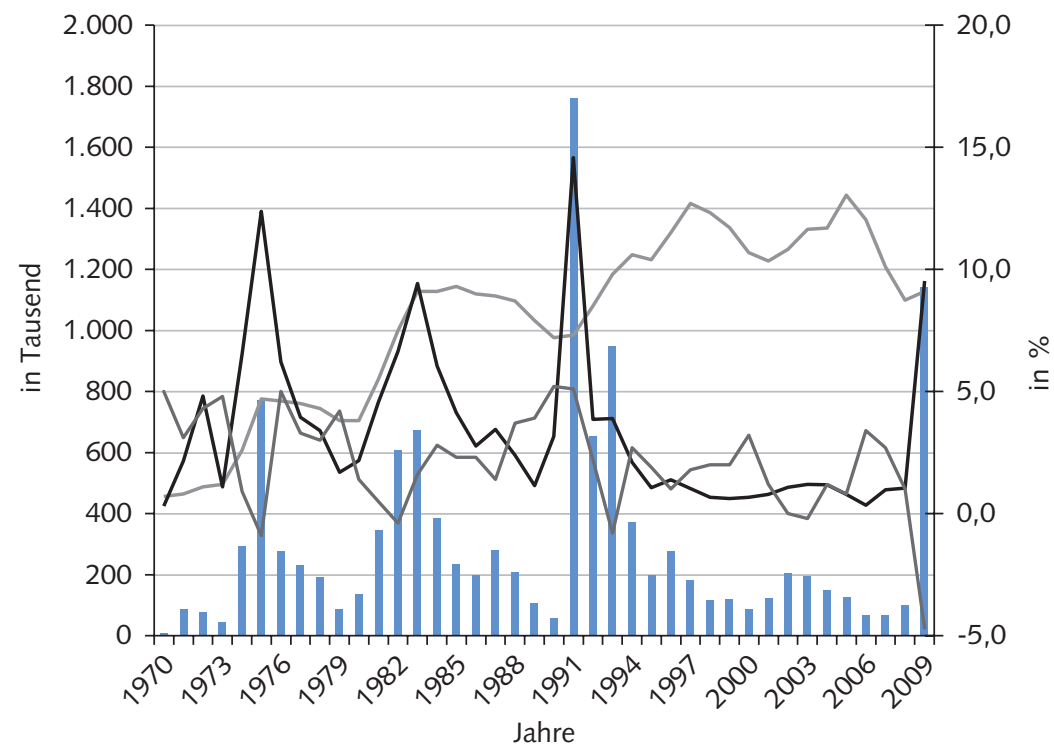

Kurzarbeitergeldempfänger
Arbeitslosenquote
Ausgabenanteil für Kurzarbeit an Gesamtausgaben der BA
BIP-Wachstum

\section{Abb. 2: Kurzarbeit im betrieblichen Kontext}

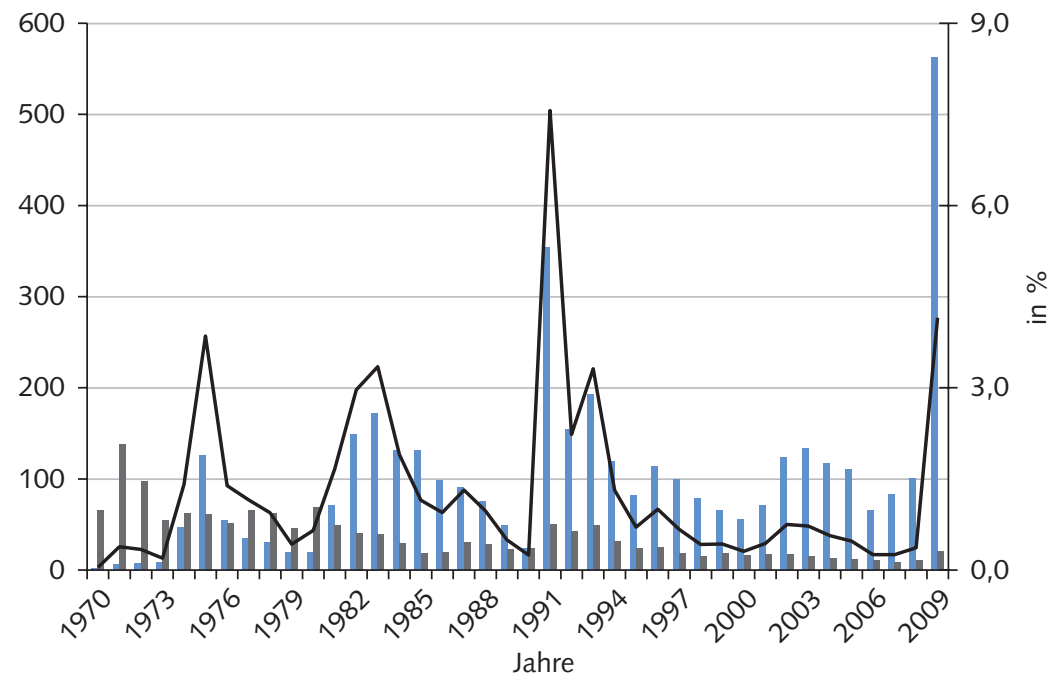

Anzahl kurzarbeitender Betriebe (in Hundert)
Kurzarbeiter pro Betrieb
Anteil Kurzarbeiter an sozialversicherungspflichtig Beschäftigten

WSI MITTEILUNGEN

den Phasen der konjunkturellen Erholung nicht gelang, die Arbeitslosigkeit bestimmter Problemgruppen abzubauen, sodass aus jedem Abschwung eine Restarbeitslo- sigkeit zurückbehalten wurde (Hysteresis) und nicht mehr das niedrigere Ausgangsniveau erreicht wurde. Die Entwicklung der Arbeitslosigkeit ergibt seit den 1970er
Jahren bis 2005 folglich ein Treppenmuster. Erst im jüngsten Aufschwung konnte dieses Muster durchbrochen werden. Inwiefern KUG daher wirklich zur Beschäftigungssicherung beigetragen hat, kann an dieser Stelle nicht weiter analysiert werden.

Abbildung 2 zeigt, dass die Anzahl der kurzarbeitenden Betriebe zu den gleichen Zeitpunkten ihre Spitzen erreicht wie die Zahl der Empfänger von KUG.

Allerdings offenbart Abbildung 2 auch einen anderen Trend: Die Zahl der Kurzarbeiter pro Betrieb hat sich im Laufe der Zeit verringert. Dies hängt u.a. mit der Nutzung des KUG durch Betriebe kleiner und mittlerer Größe (Deeke 2005) zusammen. Insbesondere ist die Zahl der kurzarbeitenden Betriebe absolut angestiegen. So nutzten 2009 im Jahresdurchschnitt 56.244 Betriebe Kurzarbeit, 1975 waren es lediglich 12.548. Diese absoluten Zahlen sind im Vergleich mit Vorsicht zu interpretieren, da sich durch die Wiedervereinigung die Grundgesamtheit sowohl der Erwerbspersonen als auch der Betriebe vergrößert hat. Die Zahl der KUG-Empfänger als Anteil an allen sozialversicherungspflichtig (SV) Beschäftigten bestätigt das oben beschriebene Muster. 1975, 1983 und 2009 sind die Spitzenjahre mit zwischen 3 und $4 \%, 1991$ empfingen sogar mehr als $7 \%$ KUG. Seit der Wiedervereinigung ist die Zahl der Betriebe in Gesamtdeutschland allerdings leicht rückläufig. Die Verteilung der Betriebsgrößenklassen hat sich dagegen im letzten Jahrzehnt als sehr stabil erwiesen. Wenn also in mehr kleinen und mittelgroßen Betriebe KUG Anwendung findet, dann muss sich der Nutzen für diese Betriebe verbessert haben.

Denn der Vergleich der Zahlen im Zeitverlauf verschleiert, dass zu den unterschiedlichen Zeitpunkten unterschiedliche Regeln für den Bezug für KUG galten. Das Muster muss folglich nicht allein als das Ergebnis konjunktureller Schwankungen interpretiert werden, sondern auch als das Resultat politischer Steuerung. Im Nachfolgenden soll mit einem Vergleich der politischen Reaktionsmuster in den unterschiedlichen Abschwüngen gezeigt werden, dass die Entwicklung der Inanspruchnahme von KUG nicht nur eine Reaktion auf funktionale Anforderungen des Arbeitsmarktes aufgrund veränderter ökonomischer und sozialer Rahmenbedingungen ist, sondern auch das Ergebnis politischer Zielvorstellungen. 


\section{Übersicht 1: Veränderungen der KUG-Regulierung in fünf Abschwüngen seit 1970}

\section{Politics matter}

Die Frage, inwiefern politische Kräfteverhältnisse und Interessenkonstellationen die Ausgestaltung von Politiken beeinflussen, gehört zu den Kernfragen der Politikwissenschaft (Castles/McKinlay 1979; Schmidt 1982). Wie oben bereits gezeigt, kann den unterschiedlichen Akteuren AN, AG und Staat - unter bestimmten Bedingungen das gemeinsame Interesse unterstellt werden, Beschäftigung zu sichern. Alle drei werden in der konkreten Situation Kosten und Nutzen abwägen. Für die weitere Analyse unterstellen wir hierfür konstante Interessen. Die AG werden immer dann KUG zur Beschäftigungssicherung nutzen, wenn dieses besonders "günstig“ zu haben ist und sich somit das Verhältnis zu den Remanenzkosten der Kurzarbeit für sie verbessert. Stellschrauben hierfür sind vor allem die Übernahme der SVBeiträge durch den Staat und die Verringerung des administrativen Aufwandes. ${ }^{3}$ Gleichfalls sind auch die Annahmen über die Kosten der Humankapitalverluste bei Entlassungen und Wiedergewinnung neuer Arbeitskräfte, wenn die Wirtschaft wieder anzieht, entscheidend. Hierbei berücksichtigen AG vorherige Erfahrungen und Erwartungen über die Gewinnung der für sie relevanten Fachkräfte. Für die AN und ihre kollektiven Vertreter sind gleichfalls die Erwartungen über die allgemeine Entwicklung der Beschäftigung in den betroffenen Branchen relevant. Sie wägen ihre Zustimmung zur Kurzarbeit gegen das Risiko der (dauerhaften) Arbeitslosigkeit und der Möglichkeit ab, zeitnah eine vergleichbare Tätigkeit wieder zu finden. ${ }^{4}$ Die finanziellen Einschränkungen der AN entsprechen zwar den Verlusten in der Arbeitslosigkeit (zumindest für die Zeit, in der Arbeitslosengeld gezahlt wird). Ihre Einbußen sind in der Kurzarbeit jedoch als geringer zu bewerten als in der Arbeitslosigkeit, da sie im Betrieb verbleiben und sich damit Zugang zu Wissen und Ressourcen bewahren. Die finanzielle Hauptlast dagegen hat der Staat zu tragen, da er den Beschäftigten zumindest anteilig ihre Einkommensverluste erstattet und darüber hinaus Ausfälle bei Steuern und Abgaben verbuchen muss. Die jeweilige Regierung muss daher abwägen, ob die Ausgabenexpansion angesichts knapper

\begin{tabular}{|c|c|}
\hline Abschwung & Veränderung der KUG-Regulierung \\
\hline I: $1973 q 2-1975 q 2$ & $\begin{array}{l}\text { Verlängerung der Bezugsdauer des KUG auf bis zu zwei Jahre } \\
\text { Erhöhung des Lohnersatzes auf } 68 \% \\
\text { Übernahme der SV-Beiträge }\end{array}$ \\
\hline II: 1979q4-1982q4 & Keine Änderung des KUG, aber arbeitsmarktpolitisches Sonderprogramm \\
\hline III: 1991q1-1993q3 & $\begin{array}{l}\text { KUG wird auch an AN gewährt, wenn der Arbeitsausfall nicht konjunkturell, } \\
\text { sondern strukturell (schwerwiegende Verschlechterung der Lage des } \\
\text { Wirtschaftszweiges) begründet ist } \\
\text { Ab } 1989 \text { und im Osten ab } 1992 \text { müssen Betriebe die SV-Beiträge für die } \\
\text { Kurzarbeitenden selbst zahlen }\end{array}$ \\
\hline IV: 2001q1-2005q2 & $\begin{array}{l}\text { Befristete Struktur-KUG-Regelungen werden bis zum 31.12.2006 verlängert, } \\
\text { ab } 2004 \text { entfristet (umbenannt in Transfer-KUG) } \\
\text { Bezugsdauer wird auf zwölf Monate erhöht zur Förderung der Eingliederung }\end{array}$ \\
\hline V: 2008q1-2009q2 & $\begin{array}{l}\text { Abschaffung Drittelregelung/Entgeltausfall mehr als } 10 \text { \% insgesamt } \\
\text { ausreichend } \\
\text { maximale Bezugsfrist auf } 24 \text { Monate verlängert } \\
\text { ab 1.7.09 Übernahme SV-Beiträge durch BA, ab dem 7. Monat, bei Qualifi- } \\
\text { zierung volle Übernahme } \\
\text { Die Notwendigkeit, zuvor auch Minusstunden aufzubauen, wurde außer Kraft } \\
\text { gesetzt } \\
\text { Erleichterungen bei der Beantragung und der Nachweispflicht der Betriebe }\end{array}$ \\
\hline
\end{tabular}

Quelle: Eigene Zusammenstellung auf Basis von Steffen 2009 und IAB 2010. WSI MITTEILUNGEN

Ressourcen zur Beschäftigungssicherung zu rechtfertigen ist. Gleichzeitig befindet sie sich in einem Dilemma: Je attraktiver sie die KUG-Regulierung für die Betriebe gestaltet, desto größer wird das Risiko, dass auch Betriebe Unterstützung erhalten, die auch auf anderen Wegen in der Lage wären, das Konjunkturtal zu überbrücken, oder deren Krise nicht allein konjunkturell begründet ist. Die Gefahr möglicher „Trittbrettfahrer" steigt vor allem dann, wenn - wie im aktuellen Abschwung geschehen - die Nachweispflicht der Voraussetzungserfüllung gelockert wird. Gleichzeitig kann den Regierungen ein großes Interesse unterstellt werden, Arbeitslosigkeit zu begrenzen, um eine mögliche Wiederwahl nicht zu gefährden.

Im Folgenden werden daher zunächst die politischen Reaktionsmuster analysiert und anschließend wird beschrieben, welche Faktoren eine Ausweitung von KUG begünstigen.

\subsection{POLITISCHE REAKTIONSMUSTER}

In Übersicht 1 sind die jeweiligen Anpassungen in Bezug auf die KUG-Regulierungen in den fünf zu vergleichenden Abschwüngen zusammengestellt. Es zeigt sich, dass das oben beschriebene Muster, wonach im jüngsten Abschwung deutlich mehr Betriebe die Kurzarbeit nutzten, mit erheblichen Modifikationen der KUG-Regulierung einherging. Durch zahlreiche Veränderungen hat das KUG deutlich an Attraktivität für Unternehmen gewonnen (Crimmann /Wießner 2009).
Die jeweils amtierenden Regierungen haben folglich die KUG-Regulierungen sehr unterschiedlich angepasst, um dem Konjunktureinbruch zu begegnen. Deutliche Verbesserungen finden sich in den Abschwüngen zu Beginn der 1970er Jahre, zur Wiedervereinigung und im aktuellen Abschwung. Im vorangegangenen Einbruch hatte das KUG dagegen kaum eine Rolle gespielt.

Mit dem Ende des „kurzen Traums immerwährender Prosperität“ (Lutz 1984) infolge der Ölkrisen in den 1970er Jahren endete eine Phase der Vollbeschäftigung und die Bekämpfung von Arbeitslosigkeit avancierte zum neuen Ziel der Politik. Die Arbeitsmarktpolitik wurde durch mehrere Sonderprogramme erweitert (Bieback 1978). Zusammen mit einer expansiven Fiskalpolitik und der Förderung von Investitionen wollte die frisch gewählte sozialliberale Koalitionsregierung unter Bundeskanzler Helmut Schmidt (1974-1976) antizyklisch den Erhalt und die Schaffung von Arbeitsplätzen begünstigen. Gleichzeitig begegnete die Regierung ab 1975 der Kostenexplosion mit Ausgabenbeschränkungen im Bereich der Unterhaltsleistungen für berufliche Weiterbildung und verschärfter Zumutbarkeit für ALG- und Arbeitslosenhilfebezieher (Bieback 1978). Dieser Kurs einer gedämpften Kostenent-

3 Erstattet wurden zunächst $50 \%$ des Beitrags für die Ausfallzeit (Bruche/Reissert 1985). Dies entfiel 1989.

4 Bei der Einführung von Kurzarbeit muss der Betriebsrat zustimmen, da damit finanzielle Einbußen der Beschäftigten verbunden sind. 
wicklung prägte auch die nachfolgenden Jahre: Während sich die Ausgaben für die monetären Lohnersatzleistungen im Falle von Arbeitslosigkeit von 1973 bis 1983 verzehnfacht hatten, hatten sich die Ausgaben der aktiven Arbeitsmarktpolitik (AAMP) lediglich verdoppelt (Bruche/Reissert 1985). Unter den aktiven Maßnahmen gehörten das KUG sowie Arbeitsbeschaffungsmaßnahmen zu den bedeutendsten (Oschmiansky/Ebach 2009). Die Zahl der KUG-Empfänger war in den Krisenjahren 1974/75 und 1982/83 besonders hoch (Flechsenhar 1979; Abbildung 1). Aufgrund der lang anhaltenden Hochkonjunkturphase waren im ersten Abschwung die Überstundenkonten reichlich gefüllt. Die Betriebe waren verpflichtet, diese zunächst abzubauen. Allerdings wurde den Betrieben der KUG-Einsatz durch die volle Erstattung der SV-Beiträge deutlich vergünstigt. Im zweiten Abschwung spielte zwar die allgemeine Arbeitszeitdebatte eine große Rolle, die Ausweitung der KUGRegulierung wie zuvor fand jedoch nicht statt. Betrieben, die trotz ihrer Anpassungs- und Umstellungsprobleme zusätzliche Bildungsmaßnahmen durchführten und so vorbeugende Arbeitsmarktpolitik betrieben, wurden 80-100 \% der Lohnkosten für von der Arbeit Freigestellten erstattet (IAB 2010).

In der besonderen Arbeitsmarktsituation nach der Wiedervereinigung, die sich aus der Transformation der neuen Bundesländer von der Plan- zur Marktwirtschaft ergab, kam das gesamte arbeitsmarktpolitische Instrumentarium extensiv zum Einsatz. Allerdings zielte die Expansion des KUG nicht auf den vorübergehenden Arbeitsausfall. Vielmehr war es Ziel der Politik, den irreversiblen Personalabbau infolge des Transformationsprozesses abzufedern. ${ }^{5}$ Man erhoffte sich einen Beitrag zur Lösung des Strukturwandels in Ostdeutschland über die Verbindung von Kurzarbeit und Qualifizierung (Seifert et al. 1993). Inwiefern sich dieser neue Anspruch an das KUG realisierte, wird in der Literatur unterschiedlich eingeschätzt. Denn der massenhafte Wegfall von Arbeitsplätzen wurde nur kurzfristig abgefedert. Die strukturellen Probleme wurden nicht gelöst und die Arbeitslosigkeit stieg stark an (Abbildung 1). Diese Ausnahmesituation scheint sich daher für die Erklärung, unter welchen Bedingungen Regierungen das KUG als Kriseninstrument intensiv nutzen, wenig zu eignen.

Interessanter erscheint die Frage, warum in dem lang anhaltenden Abschwung zu Beginn der 2000er Jahre keine Ausweitung des KUG stattfand. Denn infolge der persistent hohen Arbeitslosigkeit nach der Wiedervereinigung hatten sich Ziele, Maßnahmen und Instrumente der Arbeitsmarktpolitik deutlich verändert (Bogedan/Bothfeld/Sesselmeier 2009). Über eine Verschärfung der Zumutbarkeitsregelungen und die Ausweitung von Sanktionsmöglichkeiten sollte die Konzessionsbereitschaft der Arbeitslosen erhöht werden. Die Wiederaufnahme einer (beliebigen) Beschäftigung sollte Vorrang vor arbeitsmarktpolitischen Leistungen haben. Das KUG nimmt hinsichtlich dieser neuen arbeitsmarktpolitischen Ausrichtung eine zwiespältige Rolle ein. Einerseits steht KUG im Widerspruch zur Anreiz- und Wettbewerbslogik, da es weder für Betriebe noch für Beschäftigte ausreichend Anreize bietet, die unternehmerische Wettbewerbsbzw. individuelle Beschäftigungsfähigkeit zu verbessern. Mittelfristig können sich daher negative Effekte einstellen, wenn dadurch notwendige Strukturanpassungen unterbleiben (Brenke/Zimmermann 2010). Andererseits könnte das KUG durchaus als aktivierendes Instrument genutzt werden, das zum Erhalt der Beschäftigungs- fähigkeit beiträgt. ${ }^{6}$ Dies gilt insbesondere dann, wenn Beschäftigte im Betrieb und in Beschäftigung gehalten werden und ihnen gleichzeitig Zugang zu Qualifizierung gewährt wird. Denn je länger Arbeitslosigkeit dauert, desto einschneidender vollzieht sich eine Entwertung des Humankapitals und damit der Verfall der Beschäftigungsfähigkeit (Promberger et al. 2008). Die geringe Nutzung des KUG im vierten Abschwung dürfte - neben dem besonderen Charakter des Abschwungs ${ }^{7}$ - in einer pessimistischen Lesart des KUG begründet sein.

Die 2008 und 2009 befristet eingeführten KUG-Modifikationen sind dagegen - wie von Stein/Aricò in diesem Heft argumentiert - in eine andere beschäftigungspolitische Strategie eingebettet. Zur Dämpfung des Arbeitslosigkeitsrisikos wurden die KUG-Regulierungen so modifiziert, dass der Einsatz für Betriebe attraktiv wurde. In der gegenwärtigen Variante entstehen für die Betriebe dann die geringsten Kosten, wenn sie-wie politisch gewollt - den Arbeitsausfall auf mehrere Schultern verteilen und Kurzarbeit mit Qualifizierung verbunden wird. Diese Variante ist auch für die kurzarbeitenden Beschäftigten die „günstigste“ (Crimmann et al. 2009). Wie auch im ersten Abschwung wurde dafür an folgenden entscheidenden „Stellschrauben“ gedreht: Der Zugang zu den Leistungen wurde erleichtert, die Voraussetzungen, die Betriebe und Beschäftigte erfüllen müssen, verringert, die Lohnersatzleistungen wurden erhöht und die Kosten der Betriebe wurden durch die (teilweise) Übernahme der SV-Beiträge gesenkt.

Politische Entscheidungen bilden daher eine wichtige lenkende Kraft für den Einsatz von KUG. Fraglich ist darüber hinaus, welche Faktoren die unterschiedlichen Reaktionen in den verglichenen Abschwüngen erklären.

\section{Tabelle 1: Vergleich der Regierungskonstellationen in fünf}

\section{Abschwüngen seit 1970}

\begin{tabular}{|c|c|c|c|c|c|c|c|c|}
\hline \multirow[t]{2}{*}{ Abschwung } & \multirow[t]{2}{*}{$\begin{array}{l}\text { Regierungs- } \\
\text { koalition }\end{array}$} & \multirow{2}{*}{$\begin{array}{c}\text { Parteizuge- } \\
\text { hörigkeit } \\
\text { Kanzler/in }\end{array}$} & \multirow{2}{*}{$\begin{array}{l}\text { Parteizuge- } \\
\text { hörigkeit } \\
\text { Arbeits- } \\
\text { minister/in }\end{array}$} & \multicolumn{4}{|c|}{$\begin{array}{l}\text { Gewichtete Regierungs- } \\
\text { monate }\end{array}$} & \multirow[t]{2}{*}{$\begin{array}{l}\text { Wahl- } \\
\text { termine }\end{array}$} \\
\hline & & & & SPD & CDU & FDP & Grüne & \\
\hline I: 1973q2-1975q2 & SPD/CDU & SPD & SPD & 18,39 & & 6,61 & & \\
\hline II 1979q4-1982q4 & $\begin{array}{l}\text { SPD/CDU } \\
\text { CDU/FDP }\end{array}$ & $\begin{array}{l}\text { SPD/ } \\
\text { CDU }\end{array}$ & $\begin{array}{l}\text { SPD/ } \\
\text { CDU }\end{array}$ & 27 & 0,76 & 9,24 & & 5.10 .1980 \\
\hline III: 1991q1-1993q3 & CDU/FDP & CDU & CDU & & 23,25 & 7,75 & & \\
\hline IV: $2001 q 1-2005 q 2$ & SPD/Grüne & SPD & SPD & 41,48 & & & 10,52 & 22.09 .2002 \\
\hline V: 2008q1-2009q2 & $\begin{array}{l}\text { CDU/SPD } \\
\text { CDU/FDP }\end{array}$ & CDU & $\begin{array}{l}\text { SPD/ } \\
\text { CDU }\end{array}$ & 8,5 & 11,25 & 1,24 & & 27.09.2009 \\
\hline
\end{tabular}

5 Zahlreiche Arbeitsplätze wurden im gewerblichindustriellen und im landwirtschaftlichen Sektor abgebaut. Entsprechend rückläufig waren die Erwerbstätigenzahlen in diesen Sektoren (vgl. SVR 2004).

6 Zwar wurde immer wieder versucht, KUG mit Qualifizierung zu verbinden, doch blieb der Erfolg im jüngsten Abschwung begrenzt (Bogedan 2010).

7 Dieser Abschwung unterscheidet sich von den anderen aufgrund seiner langen Dauer und dem geringsten Einbruch im BIP. 


\subsection{PARTEIPOLITISCHE PRÄFERENZEN UND WIEDERWAHLINTERESSEN}

Dass sich Regierungsaktivitäten spürbar unterscheiden, je nachdem ob sozialdemokratische oder bürgerliche Parteien an der Macht sind (Schmidt 1982), hat die vergleichende Wohlfahrtsstaatsforschung für die Phase wohlfahrtsstaatlicher Expansion nachdrücklich gezeigt. Ein weniger eindeutiges Muster ergibt sich hingegen in den vergangenen drei Jahrzehnten (Kittel/ Obinger 2003).

Die Expansion des KUG steht im Gegensatz zu einer an der Begrenzung der Staatsausgaben orientierten Konsolidierung wohlfahrtsstaatlicher Politik. Da einige der Anpassungen der KUG-Regulierung auf dem Weg der einfachen Verordnung durch den zuständigen Minister möglich und somit relativ leicht und kurzfristig umsetzbar sind, können kurzfristige Interessen der Regierung - wie parteipolitische Präferenzen oder Wiederwahlinteressen - die Entscheidungsfindung beeinflussen.

Der Parteiendifferenzthese zufolge müsste die parteipolitische Zusammensetzung der Regierungen in den jeweiligen Abschwüngen einen mehr als zufälligen Zusammenhang mit der Entscheidung für eine Ausweitung der KUG-Regulierung aufweisen (Schmidt 1998). Vergleicht man die parteipolitische Zusammensetzung der jeweiligen Regierungen, lassen sich jedoch wenige Rückschlüsse auf solche Effekte ziehen. In vier der fünf Abschwungsphasen war die SPD an der Regierung beteiligt und stellte den zuständigen Arbeitsminister. Zur Messung des anteiligen Gewichts der jeweiligen Partei an der Regierung in den betreffenden Abschwüngen wird die Zahl ihrer Regierungsmonate mit dem Anteil ihrer Kabinettssitze gewichtet. Dabei fällt auf, dass die SPD mit 95,37 gewichteten
Regierungsmonaten im Vergleich zur CDU mit nur 35,26 am stärksten politisch in den Abschwüngen involviert war.

Der Abschwungvergleich zeigt, dass das KUG sowohl von sozial- als auch christdemokratisch geführten Regierungen expansiv modifiziert wurde. Es ist daher $\mathrm{zu}$ vermuten, dass weniger parteipolitische Präferenzen den Einsatz antrieben als die Möglichkeit, auf diesem Weg kurzfristig Arbeitslosigkeit einzudämmen.

Das Interesse an einer solchen kurzfristigen Eindämmung der Arbeitslosigkeit könnte zudem durch Wiederwahlinteressen gesteigert werden. Während allerdings die Nähe zur Bundestagswahl im letzten Abschwung einen Einfluss auf die Entscheidung der amtierenden Großen Koalition gehabt haben könnte, die Ausgaben für KUG auszuweiten, sprechen der zweite und vierte Abschwung gegen einen solchen Einfluss. Vielmehr zeigt sich, dass die starken Modifizierungen in den KUG-Regulierungen in Zeiten einer Großen Koalition zwischen CDU und SPD stattfanden.

\section{5 Konjunktur drängt, Politik lenkt}

Zusammenfassend lässt sich festhalten, dass das Muster des Instrumenteneinsatzes zwei interessante Befunde offenbart:

(1) Nicht allein der Konjunkturverlauf ist für den Einsatz des KUG verantwortlich. Zwar kann ein Konjunktureinbruch als Auslöser wirken, doch inwiefern KUG vermehrt zum Einsatz kommt, wird durch politische Entscheidungen erheblich „gelenkt“. Wenn Politik jedoch zu kurzfristigen Erfolgen neigt, um das eigene politische Überleben zu sichern (Scharpf 1986), dann wird sie die Arbeitsmarktpolitik nutzen, um „schnelle“Arbeitsmarktentlastungen zu schaffen. Nutzen und Gefahren des KUG sind folglich beide in der kurzfristigen Durch- und Umsetzungsfähigkeit begründet. Denn mit dem KUG steht der Politik ein Instrument zur Verfügung, um zeitnah Arbeitslosigkeit zu drosseln. Es nimmt somit Druck aus dem Arbeitsmarkt und schlägt bestenfalls eine Brücke über das Konjunkturtal. Gleichzeitig sind vonseiten der AG keine Widerstände gegen eine Ausweitung oder Lockerung der Regulierung des KUG zu erwarten. Eine Ausweitung des KUG liegt dabei nicht immer im Interesse aller AN, da oftmals nur eine Teilgruppe profitiert, die Kosten aber von allen über die Beitragsmittel zur BA zu tragen sind.

(2) Parteipolitische Dispositionen und das politische Kräfteverhältnis scheinen keinen starken Einfluss auf die Regulierung des KUG und des arbeitsmarktpolitischen Instrumentariums genommen $\mathrm{zu}$ haben, wenngleich für eine quantifizierende Bewertung geeignete Vergleichsmaßstäbe fehlen. Allerdings ist die Parteiendifferenzthese dann am stärksten, wenn sie nicht isoliert betrachtet wird, sondern im Zusammenhang mit anderen sozioökonomischen, machtpolitischen und institutionenbezogenen Variablen. Hierzu ist künftig weitere Forschung notwendig, die u.a. stärker die Interessen der abhängig Beschäftigten berücksichtigt. Denn angesichts eines andauernd hohen Arbeitsmarktrisikos für bestimmte Problemgruppen und des Auslaufens der Förderung einiger Instrumente im Jahr 2011 bleibt die Auseinandersetzung über Ziele und Maßnahmen einer zukünftigen Arbeitsmarktpolitik weiter aktuell. 
Bach, H.-U./Crimmann, A./Spitznagel, E./Wießner, F. (2009): Kurzarbeit: Atempause in der Krise, in: IAB-Forum Spezial, S. 52-63 Bach, H.-U./Spitznagel, E. (2009): Kurzarbeit: Betriebe zahlen mit und haben etwas davon, IAB-Kurzbericht 17, Institut für Arbeitsmarktund Berufsforschung, Nürnberg

Bieback, K.-J. (1978): Arbeitslosigkeit und Arbeitsvermittlung, in: Hochschule für Wirtschaft und Politik (Hrsg.): Arbeitsmarktpolitik, Wiesbaden, S. 82-103

Bogedan, C. (2010): Qualifizieren statt Entlassen - Betriebliche Weiterbildung in der Krise, in: WSI-Mitteilungen 6, S. 314-319

Bogedan, C./Bothfeld, S./Sesselmeier, W. (2009): Arbeitsmarktpolitik in der sozialen Marktwirtschaft - Vom Arbeitsförderungsgesetz zum Sozialgesetzbuch II und III, in: Bothfeld, S./Bogedan, C./Sesselmeier, W. (Hrsg.): Arbeitsmarktpolitik in der sozialen Marktwirtschaft. Vom Arbeitsförderungsgesetz zum Sozialgesetzbuch II und III, Wiesbaden, S. 7-18

Bogedan, C./Brehmer, W./Herzog-Stein, A. (2009): Betriebliche Beschäftigungssicherung in der Krise: Eine Kurzauswertung der WSIBetriebsrätebefragung 2009, WSI Report 1

Brenke, K./Zimmermann, K. F. (2010): Kurzarbeit: Nützlich in der Krise, aber nun den Ausstieg einleiten, in: DIW-Wochenbericht 16, S. 2-13 Bruche, G./Reissert, B. (1985): Die Finanzierung der Arbeitsmarktpolitik: System, Effektivität, Reformansätze, Frankfurt/New York

Castles, F./McKinlay, R. D. (1979): Does Politics Matter? An Analysis of the Public Welfare Commitment in Advanced Democratic States, in: European Journal of Political Research 2, S. 169-186

Crimmann, A./Möller, J./Stops, M./Walwei, U. (2009): Kurzarbeit. Wann und wie lange lohnt sie sich?, in: IAB-Forum 2, S. 104-111

Crimmann, A./Wießner, F. (2009): Wirtschafts- und Finanzkrise: Verschnaufpause dank Kurzarbeit, IAB-Kurzbericht, Institut für Arbeitsmarkt- und Berufsforschung, Nürnberg

Deeke, A. (2005): Kurzarbeit als Instrument betrieblicher Flexibilität. Ergebnisse aus dem IAB-Betriebspanel 2003, IAB-Forschungsbericht 12, Institut für Arbeitsmarkt- und Berufsforschung, Nürnberg

Eichhorst, W./Marx, P. (2009): Kurzarbeit: Sinnvoller Konjunkturpuffer oder verlängertes Arbeitslosengeld?, in: Wirtschaftsdienst 5,

S. 322-328

Flechsenhar, H.-R. (1978): Kurzarbeit - Kosten und Finanzierung, in: Mitteilungen aus der Arbeitsmarkt- und Berufsforschung (MittAB) 4, S. 443-456

Flechsenhar, H.-R. (1979): Kurzarbeit - Strukturen und Beschäftigungswirkung, in: Mitteilungen aus der Arbeitsmarkt- und Berufsforschung (MittAB) 12, S. 362-372

Haag, O./Discher, M. (2002): Kurzarbeit - Alternative zur Kündigung?, in: Arbeit und Arbeitsrecht 11, S. 494-498

Heckmann, M./Kettner, A./Pausch, S./Szameitat, J./Vogler-Ludwig, K. (2009): Wie Betriebe in der Krise Beschäftigung stützen, IAB-Kurzbericht 18, Institut für Arbeitsmarkt- und Berufsforschung, Nürnberg
Herzog-Stein, A./Seifert, H. (2010): Stabile Beschäftigung durch flexible Arbeitszeiten, WSI-Diskussionspapier 169

Institut für Arbeitsmarkt- und Berufsforschung (IAB) (2010):

Arbeitsmarktpolitisches Informationssystem, http://www.iab.de/de/ informationsservice/informationssysteme/arbeitsmarktpolitik.aspx (01.09.2010)

Kittel, B./Obinger, H. (2003): Political Parties, Institutions and the Dynamics of Social Expenditure in Times of Austerity, in: Journal of European Public Policy 1, S. 20-45

Lutz, B. (1984): Der kurze Traum immerwährender Prosperität: Eine Neuinterpretation der industriell-kapitalistischen Entwicklung im Europa des 20. Jahrhunderts, Frankfurt/Main

Mosley, H./Kruppe, T. (1995): Kurzarbeit im Strukturwandel: Europäische Erfahrungen, in: WSI-Mitteilungen 7, S. 451-462

Oschmiansky, F./Ebach, M. (2009): Vom AFG 1969 zur Instrumentenreform 2009: Der Wandel des arbeitsmarktpolitischen Instrumentariums, in: Bothfeld, S./Sesselmeier, W./Bogedan, C. (Hrsg.): Arbeitsmarktpolitik in der sozialen Marktwirtschaft. Vom Arbeitsförderungsgesetz zum Sozialgesetzbuch II und III, Wiesbaden, S. 79-93

Promberger, M./Wenzel, U./Pfeiffer, S./Hacket, A./Hirseland, A. (2008): Beschäftigungsfähigkeit, Arbeitsvermögen und Arbeitslosigkeit, in: WSI-Mitteilungen 2, S. 70-76

Sachverständigenrat zur Begutachtung der gesamtwirtschaftlichen Entwicklung (SVR) (2004): Erfolge im Ausland - Herausforderungen im Inland, Jahresgutachten 2004/2005, Wiesbaden

Sachverständigenrat zur Begutachtung der gesamtwirtschaftlichen Entwicklung (SVR) (2007): Das Erreichte nicht verspielen, Jahresgutachten 2007/2008, Wiesbaden

Scharpf, F. (1986): Policy failure and institutional reform: why should form follow function?, in: International Social Science Journal 108, S. 179-189

Schmid, G./Semlinger, K. (1980): Instrumente gezielter Arbeitsmarktpolitik: Kurzarbeit, Einarbeitungszuschüsse, Eingliederungsbeihilfen, Königstein/Taunus

Schmidt, M. G. (1982): Wohlfahrtsstaatliche Politik unter bürgerlichen und sozialdemokratische Regierungen. Ein internationaler Vergleich, Frankfurt/New York

Schmidt, M. G. (1998): Sozialpolitik in Deutschland. Historische Entwicklung und internationaler Vergleich, Opladen

Seifert, H./Ochs, C./Besselmann, K./Machalowski, G. (1993): Kurzarbeit und Qualifizierung - Bedingungen und Gestaltungsmöglichkeiten der Kurzarbeit zur Nutzung der beruflichen Qualifizierung, Forschungsbericht hrsg. vom Bundesministerium für Arbeit und Soziales, Düsseldorf/Köln

Steffen, J. (2009): Sozialpolitische Chronik. Die wesentlichen Änderungen in der Arbeitslosenversicherung, hrsg. von der Arbeitnehmerkammer Bremen 